\title{
Effect of confinement on the mode dynamics of dipole clusters
}

\begin{abstract}
André Schella, $\dagger^{\star a}$ André Melzer, ${ }^{a}$ Christoph July ${ }^{b}$ and Clemens Bechinger ${ }^{b}$
Dynamical properties of colloidal clusters composed of paramagnetic beads are presented. The clusters were trapped either in a parabolic trough or in a hard-wall confinement. In order to access the dynamics of the ensembles, the instantaneous normal mode (INM) approach is utilized, which uses cluster configurations as an input. The peaks in the mode spectra weaken when the system size is increased and when the coupling strength is lowered. The short-time diffusive properties of the clusters are deduced using the INM technique. It is found that angular diffusion is always larger than radial diffusion regardless of the shape of the external trap. Further, short-time diffusion seems to be almost independent of the coupling strength in the solid regime, but decreases with increasing packing fraction and size of the ensembles. In general, it is found that diffusion is larger for parabolically confined than for hard-wall trapped clusters.
\end{abstract}

\section{Introduction}

Colloidal systems allow the study of diverse physical processes on the "atomistic" level by direct imaging of the individual beads. ${ }^{1,2}$ Considerable progress has been made in studying for instance the transport properties in colloidal systems, ${ }^{3,4}$ the phase behaviour of colloidal monolayers, ${ }^{58}$ and the mode properties of extended suspensions. ${ }^{6,9} 11$

Beside these advances made with large ensembles, finite systems have raised immense interest in various field of physics during the past decades, whereas studying finite colloidal systems has just started recently. ${ }^{12}{ }^{23}$ In particular, they provide useful insights since they bridge the gap between single particles and extended systems, therefore offering the possibility to investigate the interplay between the particle interaction and the systems' boundary.

Dynamical information of the observed ensembles can be revealed using mode analysis techniques. The normal modes of colloidal crystals were obtained from nearest neighbour fluctuations, ${ }^{9}$ the displacement correlation matrix ${ }^{10,11}$ or via harmonic approximation. ${ }^{6}$ However, in experiments, modes are massively damped due to the viscous coupling of the colloidal particles to the surrounding media, making an interpretation rather sophisticated. Alternatively, the normal modes of parabolically and hard-wall confined clusters were analysed by Kong et al. for various types of interaction by means of simulations. ${ }^{\mathbf{1 4 , 1 5}}$

${ }^{a}$ Institute of Physics, Ernst Moritz Arndt University Greifwald, Felix Hausdorff Str. 6, 17489 Greifswald, Germany. E mail: andre.schella@ds.mpg.de

${ }^{b}$ II. Institute of Physics, Pfaffenwaldring 57, 70569 Stuttgart, Germany

$\dagger$ Present address of A. S. is MPI DS Gottingen.
A common approach to calculate the diffusion coefficient of colloidal systems is studying the mean square displacement (MSD). ${ }^{3,4,23}{ }^{26}$ However, for finite systems, calculating the MSD has its difficulties, since diffusive processes can become time dependent and crucially influenced by the external confinement. ${ }^{27,28}$ Beside that, Hunter et al. have shown that the mobility of the particles in a confined colloidal system strongly depends on the packing fraction of the colloidal clusters and the physical properties of the boundary. ${ }^{23}$

In this paper, we present a method that connects the mode properties of the system with the diffusion coefficient. In particular, we make use of the so-called instantaneous normal mode (INM) technique. ${ }^{29}{ }^{31}$ The INM approach has been extensively used to study extended liquid-like Lennard-Jones (LJ)-systems and supercooled water, ${ }^{32}{ }^{36}$ to probe the dynamics of LJ-clusters $^{30,37}$ or even to analyse DNA. ${ }^{38}$ In INM, it is argued that liquids show solid-like behaviour on short time scales and hence the transport coefficient can be deduced from INM using momentary "instantaneous" particle configurations as an input. ${ }^{29}$

Recently, the INM technique has been successfully applied to analyse the diffusive properties of finite $2 \mathrm{D}$ and $3 \mathrm{D}$ dust clusters in plasmas. ${ }^{391}$ Here, we adopt the INM technique to reveal mode properties of finite colloidal clusters by making use of momentary cluster configurations, only. Thus, INM provides an alternative access in studying the dynamics of finite colloidal suspensions. Additionally, subsequent analysis of the INM mode spectra allows to gain information about the transport properties of finite ensembles were usually direct calculation via MSD provides insufficient conclusions. The impact of different types of confinement onto the cluster dynamics will be addressed by confining particles either in parabolic or hard-wall traps. 


\section{Physical properties of dipole clusters}

The phase behaviour of a dipole system consisting of $N$ identical paramagnetic beads is completely described by the dimensionless coupling parameter ${ }^{6,8}$

$$
\Gamma=\frac{\mu_{0} M^{2}}{4 \pi b_{\mathrm{ws}}^{3}} \frac{1}{k_{\mathrm{B}} T}
$$

with $\mu_{0}$ and $M$ being the magnetic field constant and magnetization, $b_{\mathrm{WS}}=1 / \sqrt{ } \pi n$ is the Wigner-Seitz distance in $2 \mathrm{D}$ at particle density $n$ and $k_{\mathrm{B}} T$ the thermal energy of the system with $T=295 \mathrm{~K}$, in our case. Even though it is peculiar to assign a coupling parameter for finite systems, experiments have shown that $\Gamma$ can still serve as a good measure even in the case of small particle numbers. ${ }^{\mathbf{2}}$

In the experiments presented here, the whole sample cell is embedded in between a Helmholtz arrangement with the sample being in the centre of the Helmholtz coils. Thus, the interaction between the paramagnetic particles can be tuned using magnetic fields. In particular, the relation given by Blickle et al. ${ }^{42} M(B)=M_{0}[\operatorname{coth}(\alpha B) \quad 1 /(\alpha B)]$, was used to describe the magnetization $M$ as a function of the magnetic field $B$ of the Helmholtz coils $\left(M_{0}=1.204 \times 10^{-12} \mathrm{~A} \mathrm{~m}^{2}, \alpha=76 \mathrm{~T}^{-1}\right.$ are the parameters for our beads). Thus, by varying $B$, one can tune the coupling parameter of the colloidal cluster via $M$, see eqn (1). Typically, the magnetic field $B$ was controlled precisely between $10 \mathrm{mT}$ as a lower limit and $55 \mathrm{mT}$ as a higher limit.

The Hamiltonian of a $N$ particle cluster with dipole interaction is given as ${ }^{\mathbf{1 3 , 1 5}}$

$$
E=\sum_{i<j}^{N} \frac{\mu_{0} M^{2}}{4 \pi r_{i j}{ }^{3}}+V_{\mathrm{ext}}\left(\vec{r}_{i}\right),
$$

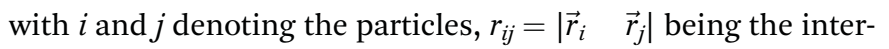
particle distance and $\vec{r}_{i}$ being the distance to the trap centre. The second term $V_{\text {ext }}$ is the external confining potential. Various types of confining geometries exist, covering the full range from circular or elliptical geometries and different functional forms ${ }^{12,16,43}$ to rectangular or even pentagonal confinement..$^{20,44}$ Here, the particles were confined either in parabolic traps

$$
V_{\mathrm{ext}}^{\mathrm{para}}=\frac{1}{2} m \omega_{0}^{2} \sum_{i} r_{i}^{2}
$$

with $m$ and $\omega_{0}$ being the particles' mass and the frequency of the confining potential, or via a hard-wall confinement

$$
V_{\mathrm{ext}}^{\mathrm{hw}}=\left\{\begin{array}{ll}
0 & \text { if } r \leq R \\
\infty & \text { if } r>R
\end{array},\right.
$$

where $R$ is the size of the hard-wall compartment. As can be seen from the different types of confinement, different length scales are needed to appropriately describe the trapped ensembles. Here, we use the normalization factors given by Kong et al. ${ }^{15}$ i.e. $r_{0}=\left(\mu_{0} M^{2} / 4 \pi\right)^{1 / 5}\left(m \omega_{0}^{2} / 2\right)^{-1 / 5}$ in the case of parabolic traps and $r_{0}=R$ as the characteristic length scale in the case of hard-wall confinement. Additionally, different confinements result in different frequency scales to describe the spectral properties of the finite ensembles. For clusters in parabolic traps, the frequencies are simply normalized to the frequency of the confining potential $\omega_{0}$, whereas the frequencies of the hard-wall confined clusters are given in units of $\omega_{0}=\sqrt{ } \mu_{0} M^{2} /\left(4 \pi m R^{5} N\right)$, which is a slightly modified version of the normalization presented by ref. 15 . To get a sense of the characteristic frequency scales, numerical estimates for the confining frequency for two clusters discussed in detail in Section 5 will be given. For a cluster consisting of $N=105$ particles confined at a magnetic field strength of $B=15 \mathrm{mT}$ and in a hard-wall compartment with a radius of roughly $R=41.3 \mu \mathrm{m}$ one finds a typical frequency scale of about $\omega_{0}=4.3 \mathrm{~s}^{-1}$. Using the definition of $r_{0}$ as the equilibrium distance of two dipole - interacting particles in a parabolic trap, one gets $\omega_{0}=2.3 \times 10^{3} \mathrm{~s}^{-1}$ for the parabolically trapped $N=71$ dipole cluster at $B=25 \mathrm{mT}^{13,45}$

\section{Experiment}

To create potential traps for colloidal particles, glass microscope slides have been spin coated with SU8 2007 before exposing the slides with UV light which hardens the thin SU8layer. Hard-wall confinement was achieved by placing a photo mask directly on top of the unhardened SU8-layer before UV exposure. To obtain a harmonic trough, a spacer was placed between mask and coating before the UV exposure, thus leading to diffuse shadowing at the mask edges. In both manners, compartments of different shape and size (ranging between 50 and $135 \mu \mathrm{m}$ ) have been generated (see Fig. 1 and 2). The spinning speed controls the thickness of the layer and thus the depth of the potential traps. Here, the depth of the hard-wall compartments was adjusted to roughly $8 \mu \mathrm{m} .{ }^{46}$ This is much larger than the gravitational length of the particles ${ }^{2,25}$ and validates the assumption of "hard-wall" traps. For the parabolic traps, a thin and curved SU8-layer remains after UV exposure.

The colloidal suspension consists of paramagnetic particles of $2 a=4.5 \mu \mathrm{m}$ size (Dyna Beads, $m=7.63 \times 10^{-14} \mathrm{~kg}$ ) ${ }^{47}$ in $2.24 \mathrm{~g} \mathrm{l}^{-1}$ SDS solution. Typically, 25-30 $\mu$ l solution were pipetted onto the sample. A cover slide protects the sample from the environment and the samples equilibrated within a few minutes. To prevent drift motion due to tilting, each sample
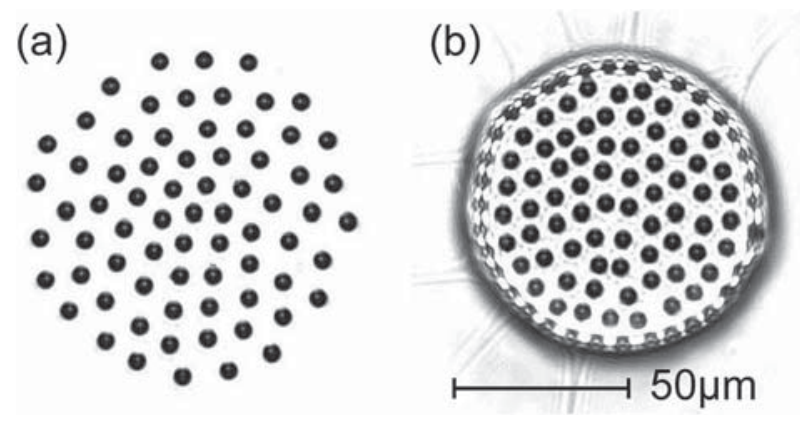

Fig. 1 Still images of (a) a parabolically confined $N \quad 71$ cluster and (b) of a $N$ 105(70) cluster in hard-wall confinement. The number of the inner particles which are not attached to the walls is given in parentheses. 

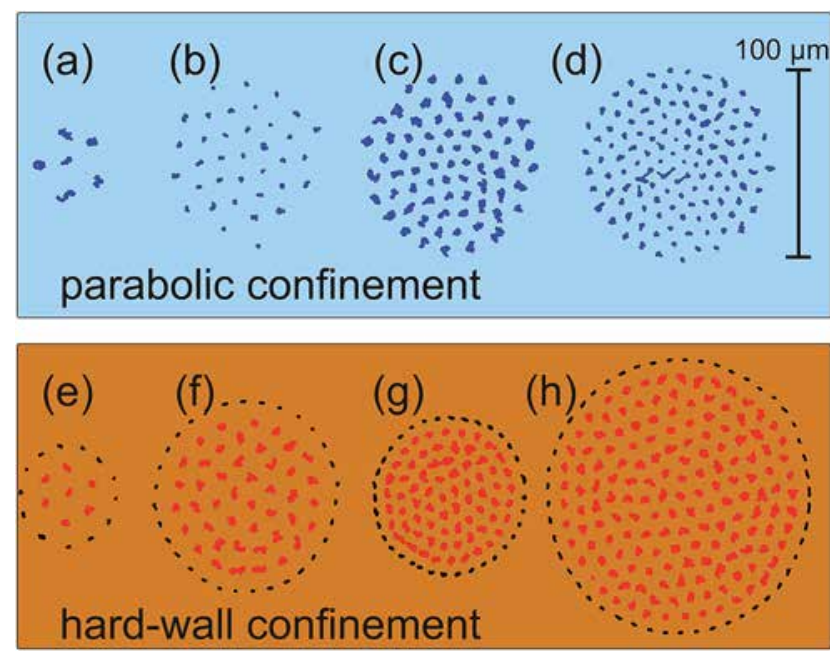

Fig. 2 Examples of reconstructed particle trajectories over a time span of 240 seconds. (a d) Particle trajectories of parabolically confined clusters and (e h) particle trajectories of hard-wall trapped clusters. (a)

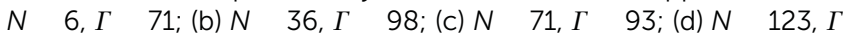

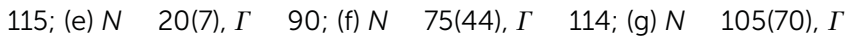
80; (h) $N \quad 204(154) ; \Gamma \quad 81$. In (e h), the particles attached to the wall are coloured in black and the number of the inner particles is given in parentheses. These wall particles are neglected for the analysis of the dynamical properties.

has been carefully adjusted. ${ }^{12}$ Particles sediment into the traps and, depending on the local density of the suspended particles and on the shape of the confinement, formed finite 2D monolayers as can be seen from the still images in Fig. 1(a) and (b). The number of trapped particles varies statistically from compartment to compartment and with the size of the traps. ${ }^{12}$ The colloidal clusters were imaged by a CCD camera connected to a microscope and the beads were traced individually using standard routines. ${ }^{48}$ The frame rate of the camera was chosen to be in the range $8-12.5 \mathrm{~Hz}$, high enough to capture the diffusive behaviour of the particles. ${ }^{1,2,49}$

For our analysis, clusters with different particle numbers and in different external confinement were realized. To achieve good statistics, we performed long run experiments with about 12000 frames per sample for the parabolically trapped clusters and 6000 frames per experiment for the hard-wall confined ensembles. The interaction between the beads was tuned via the magnetic field generated by the Helmholtz coils as described above.

Fig. 2 depicts reconstructed trajectories over a time span of 240 seconds for parabolically confined (a-d) and hard-wall trapped $(\mathrm{e}-\mathrm{h})$ colloidal clusters of various particle numbers and coupling strength. The particles perform localized Brownian motion in the potential cage created by the neighbouring particles and the external confinement. To distinguish inner particles and particles attached to the wall in the case of hardwall confinement, the trajectories of mobile inner particles are coloured in red whereas the trajectories of the outer particles are coloured in black. Here and henceforth, the number of inner particles of the hard-wall confined clusters will be given in parentheses. A mode analysis of the particles attached to the wall would yield non-physical, infinite eigenmodes. ${ }^{15}$ However, these particles contribute to the particle-particle interaction term of eqn (2) for all inner particles. Consequently, the interaction of the $i$-th inner particle is influenced by all - including the wall particles - other particles. Hence, for our mode analysis, the interaction of all $N$ cluster particles is taken into account when calculating the modes of each particle but the eigenmodes of the wall particles were excluded for our further analysis.

\section{Instantaneous normal modes}

Starting point of the INM analysis is the $2 N \times 2 N$ dynamical matrix, i.e. the second derivative of eqn (2),

$$
H(t)=\left.\frac{\partial^{2} E(\vec{r}, t)}{\partial r_{\alpha, i} \partial r_{\beta, j}}\right|_{\vec{r}(t)},
$$

which has to be calculated at each instant of time using momentary particle configurations $\vec{r}(t)$. Then, a momentary set of the $2 N$ eigenvectors and eigenvalues $e_{i, l}(t)$ and $\lambda_{l}$ of each eigenmode $l$ can be governed by solving the corresponding, instant eigenvalue problem of eqn (5). Due to the polar symmetry, it is convenient to calculate the eigenmodes of the system in polar coordinates by applying the transformation $(x, y) \rightarrow(r, \theta) \cdot{ }^{14,15}$ However, one must bear in mind that the choice of a set of coordinates has a profound influence on the INM eigenvalues. ${ }^{\mathbf{5 0 , 5 1}}$

To achieve a density of states (DOS) of the ensemble, the eigenfrequencies $\omega_{l}=\sqrt{ } \lambda_{l}$ are summed up along the time series

$$
\rho(\omega)=\left\langle\sum_{l} \delta\left(\begin{array}{ll}
\omega & \omega_{l}
\end{array}\right)\right\rangle .
$$

The density of states has to be normalized accordingly $\frac{1}{\omega_{0}} \int \rho(\omega) \mathrm{d} \omega=1$. The eigenfrequencies can be either purely real or purely imaginary, depending on the sign of the eigenvalues. Real eigenfrequencies describe the solid properties in the system, for instance when a particle oscillates in the potential cage of the nearest neighbour. ${ }^{52}$ Imaginary eigenfrequencies describe transient hopping events from one stable cluster configuration into an other. They mainly allow to deduce the fluid properties of the ensemble. ${ }^{33}{ }^{35}$ Thus, the DOS consists of two parts, $\rho(\omega)=\rho_{\mathrm{s}}(\omega)+\rho_{\mathrm{u}}(\omega)$ denoting the stable and unstable modes, respectively. The frequency of the hopping events can be quantitatively described by the hopping rate

$$
\tau_{\mathrm{h}}{ }^{-1}=c \int \frac{\omega}{2 \pi} \frac{\rho_{\mathrm{u}}(\omega)}{\rho_{\mathrm{s}}(\omega)} \rho(\omega) \mathrm{d} \omega,
$$

where the parameter $c \approx 3$ is used..$^{39,40}$ Different models exist to approximate the fraction $\rho_{\mathrm{u}}(\omega) / \rho_{\mathrm{s}}(\omega) \cdot{ }^{32,33}$ Here, we have calculated the hopping rate directly from eqn (7). Finally, one can obtain the diffusion coefficient $D$ using the INM technique by

$$
D=\frac{k_{\mathrm{B}} T}{m} \int \mathrm{d} \omega \rho(\omega) \frac{\tau_{\mathrm{h}}}{1+\tau_{\mathrm{h}}{ }^{2} \omega^{2}} .
$$


Since the INM technique only relies on the momentary particle configurations and neglects effects of the viscosity of the surrounding media, the calculated diffusion coefficients can be interpreted as the diffusion of the corresponding frictionless system. Changes in the particle configurations are captured on a time scale given by the frame rate of the camera, which is of the order of $10 \mathrm{~Hz}$ an hence on the scale of shorttime diffusive motion., ${ }^{3,24,49}$

We have performed the INM analysis to obtain the density of states for various clusters at different coupling strength and in hard-wall and parabolic confinement. Typical INM density of states are shown in Fig. 3 for the dipole clusters with comparable coupling strengths highlighted in Fig. 2 representing small $N \leq 16$, medium $16<N<50$ and large clusters $N>50$. The DOS are given in units of the normalized frequency and the unstable mode branch is plotted onto the negative frequency axis by convention. The number of freely moving particles in the hard-wall confinement is approximately that of the corresponding cluster in the parabolic case. Hence, small, medium and large clusters in different confinement should be directly compared to each other. It should be noted, that the $x$-axis is stretched by a constant factor for the small clusters as marked in Fig. 3. As already mentioned, the interaction of an arbitrary inner particle in the hard-wall compartment depends on all other particles, and hence, the dynamical matrix and the modes were computed using all particles but the modes of the particles located at the edges of the hard-wall were excluded for our subsequent INM analysis.

Let us start with the small $N=6$ parabolically and $N=20(7)$ hard-wall confined cluster, see upper row in Fig. 3(a) and (b) and

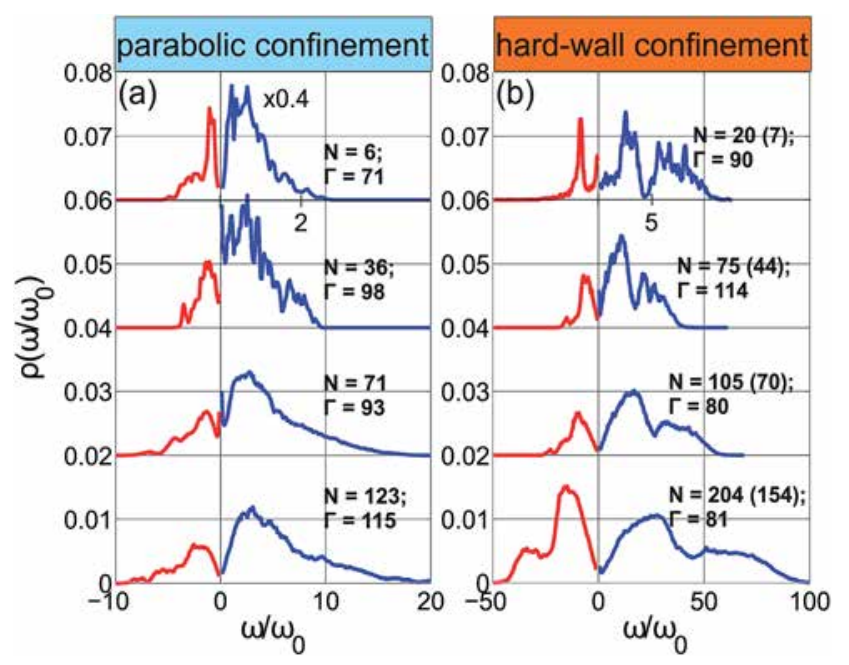

Fig. 3 (a) INM density of states of the parabolically confined colloidal clusters and (b) INM density of states of the hard-wall confined colloidal clusters highlighted in Fig. 2. Real eigenfrequencies are plotted on the positive frequency axis, imaginary eigenfrequencies are plotted on the negative frequency axis. The frequency axis was stretched by a factor of five for the $N \quad 6$ and by a factor of 2 for the $N$

20 (7) cluster. For the clusters in hard-wall confinement, the number of the inner particles is given in parentheses. Increasing the size of the system leads to diminishing of mode peaks in the density of states. See text for details.
2. Both clusters have a coupling parameter close to $\Gamma \approx 70$ and $\Gamma \approx 90$, which is above the melting point of an extended dipole interacting colloidal system $\left(\Gamma_{\mathrm{c}} \approx 61\right) ., 5$ The DOS shows distinct, sharp peaks in the stable as well as in the unstable branch for both clusters. This is due to the finite size of the ensembles, where discrete modes are excited instead of bulk phonons. ${ }^{37}$ In INM, the position of the peaks are defined by the equilibrium configurations, whereas the width of the peaks are a closely related to the particle fluctuations. ${ }^{53}$ In case of hardwall confinement $N=20(7)$, stable modes are excited up to approximately $\omega / \omega_{0}=10$. Moreover, two domains at low and high frequency are clearly separated at $\omega / \omega_{0} \approx 4$ in the stable part of the DOS. This is different for the parabolic confinement where stable modes are grouped in a single domain with only a small dip between the low and the high frequency modes. The overall fraction of unstable modes, defined by $f_{\mathrm{u}}=\int_{0}^{-\infty} \rho_{\mathrm{u}}(\omega) \mathrm{d} \omega$, is roughly $25 \%$ for the parabolic $N=6$ cluster. For the $N=20(7)$ hard-wall cluster one finds $f_{\mathrm{u}}=0.19$. These fractions are decisively larger than the unstable mode fractions of finite 2D dust clusters in plasmas, ${ }^{39,40}$ but comparable to 3D Lennard-Jones clusters ${ }^{37}$ and finite 3D dust clouds. ${ }^{41}$

The corresponding DOS of the medium-sized $N=36$ parabolically and $N=75(44)$ hard-wall confined clusters that is shown in the second row in Fig. 3(a) and (b) has still peaked features representing discrete eigenmodes. However, the peaks are not as pronounced as for the smaller systems. The stable DOS of the hard-wall confined $N=75$ (44) cluster has again two groups of high and low frequency modes with their maxima at a normalized frequency of about 11 and 22, whereas the parabolically trapped $N=36$ dipole cluster has no gap between stable high and low frequency modes. For both clusters, the fraction of unstable modes is found to be about $f_{\mathrm{u}}=0.19$.

For the larger clusters, $N=71$ and in case of parabolic confinement and $N=105(70)$ in case of hard-wall trapping, both density of states have no sharp peaks any more. The mode spectra of the $N=71$ dipole cluster in parabolic confinement is almost structureless and reminds of the density of states of an extended Lennard-Jones liquid, compare ref. 34. Contrary, one finds a splitting of low and high frequency modes with their maxima at $\omega / \omega_{0}=17$ and 32 in the stable DOS of the hard-wall trapped $N=105(70)$ cluster. The gap between the stable low frequency and high frequency modes for the $N=105(70)$ hardwall cluster is not as prominent as for the smaller hard-wall confined clusters. The fraction of unstable modes for these medium sized clusters are found as $f_{\mathrm{u}}=0.22(0.20)$ for the parabolically (hard-wall) confined $N=71(N=105(70))$ dipole system. Since the DOS is not peaked and continuous, it seems that the mode spectra already resembles the spectral properties of an extended bulk system. ${ }^{6,38}$

For the largest analysed clusters, stable modes are excited up to even higher frequencies of $\omega / \omega_{0}=100$ for the hard-wall trapped $N=204(154)$ cluster. Like for the parabolically trapped $N=71$ cluster, the mode spectra of the $N=123$ dipole cluster in parabolic confinement is again structureless. For comparison, the density of states of the $N=204(154)$ hard-wall cluster has still a low and a high frequency domain, both for the stable and 
the unstable mode branch. The fraction of unstable modes for this cluster was found to be $f_{\mathrm{u}}=0.5$, whereas $f_{\mathrm{u}}=0.22$ is revealed for the parabolically trapped $N=123$ cluster. Continuous spectra confirm previous results on large Coulomb clusters in parabolic traps. There, it has been revealed that discrete normal modes coincide with continuous hydrodynamic modes as the system size increases. ${ }^{54}$

It can be stated that increasing the number of particles leads to the diminishing of a peaked mode structure. Thus, the spectral characteristics reflect the evolution from the excitation of discrete eigenmodes to bulk-like modes. In the case of hardwall confinement, the eigenmodes are grouped in a low frequency and a high frequency domain regardless of the particle number of the cluster. However, calculating the instantaneous normal modes from the full Hessian (eqn (5)) does not allow to decide whether the specific eigenmodes have a pronounced radial or angular character.

Before focussing to radial and angular modes in detail, we aim to address whether the modes have a pronounced compression-like or shear-like character. A good measure for finite systems is given by the divergence and vorticity created by the field of eigenvectors. ${ }^{55}$ Following e.g. ref. 15 and 40, we calculated the local divergency and vorticity in the field of eigenmode $l$ as

$$
\begin{aligned}
& \Psi_{\mathrm{c}}(l)=\sum_{i=1}^{N} \Psi_{\mathrm{c}, i}{ }^{2}(l), \\
& \Psi_{\mathrm{s}}(l)=\sum_{i=1}^{N} \Psi_{\mathrm{s}, i}{ }^{2}(l),
\end{aligned}
$$

where the contribution of the $i$-th particle surrounded by $M$ neighbours is given by

$$
\begin{aligned}
& \Psi_{\mathrm{c}, i}(l)=\sum_{j=1}^{M}\left(\begin{array}{ll}
\vec{r}_{i} & \vec{r}_{j}
\end{array}\right)\left(\begin{array}{ll}
\vec{e}_{i, l} & \vec{e}_{j, l}
\end{array}\right) /\left|\begin{array}{ll}
\vec{r}_{i} & \vec{r}_{j}
\end{array}\right|^{2}, \\
& \Psi_{\mathrm{s}, i}(l)=\sum_{j=1}^{M}\left(\begin{array}{ll}
\vec{r}_{i} & \vec{r}_{j}
\end{array}\right) \times\left(\begin{array}{ll}
\vec{e}_{i, l} & \vec{e}_{j, l}
\end{array}\right) /\left|\begin{array}{ll}
\vec{r}_{i} & \vec{r}_{j}
\end{array}\right|^{2} .
\end{aligned}
$$

Fig. 4 shows the relative abundance of compressional $\Psi_{\mathrm{c}}$ and shear contribution $\Psi_{\mathrm{s}}$ as a function of the INM frequencies calculated from all INM modes for the parabolically trapped $N=$ $71(\Gamma=93)$ and the hard-wall confined $N=105(50)$ dipole cluster $(\Gamma=80)$ shown in Fig. 1 and 3. Again, only the inner, mobile particles of the hard-wall trapped cluster were taken into account for the calculation of $\Psi_{\mathrm{c}}$ and $\Psi_{\mathrm{s} \cdot}{ }^{15}$ Both histograms show qualitatively the same behaviour. Significant higher contributions are found mainly for $\Psi_{\mathrm{c}}$ for both clusters, whereas the modes do not show strong shear-like contributions in $\Psi_{\mathrm{s}}$ at all. A gap in $\Psi_{\mathrm{c}}$ is found for the parabolically confined dipole cluster at $\omega / \omega_{0}=8 \mathrm{~Hz}$. Most of the previous calculations for 2D systems focussed on frictionless simulations or weakly damped dust clusters were it was found that compressional modes are excited at higher frequencies than shear-like modes. ${ }^{15,40,55,56}$ Here, damping effects lead to the blurred histograms of $\Psi_{\mathrm{c}}$ and $\Psi_{\mathrm{s}}$ and it seems as if a more complicated motion of the particles in the field of eigenvectors is involved
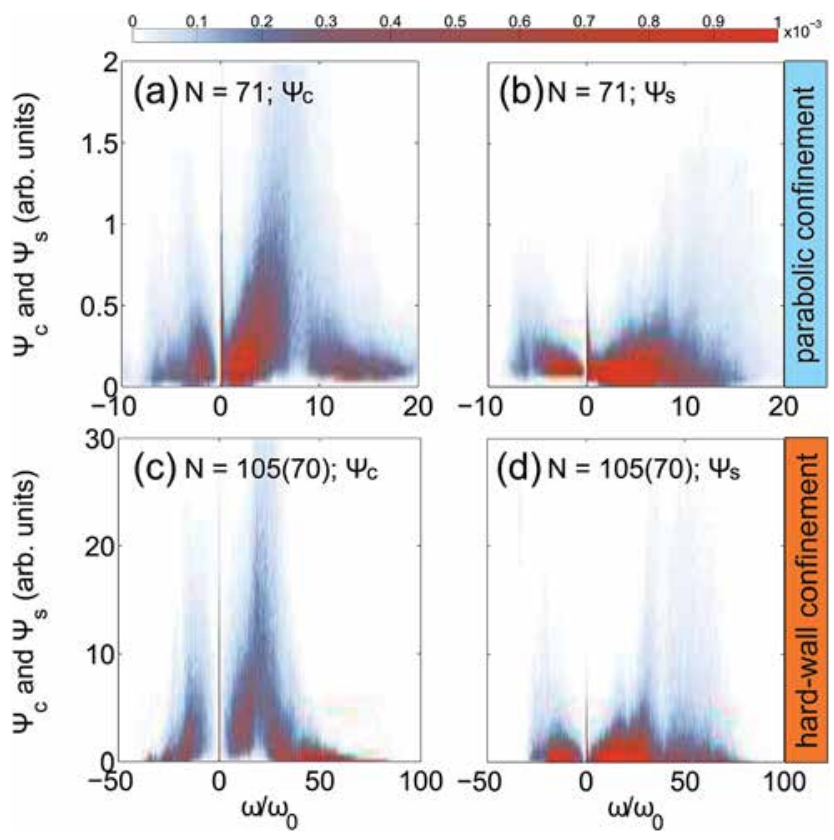

Fig. 4 Histogram of compressional $\Psi_{\mathrm{C}}(\mathrm{a})$ and shear $\Psi_{\mathrm{s}}$ contribution (b) of the INM modes of the parabolically trapped $N \quad 71$ dipole cluster ( $\left.\begin{array}{l}\Gamma \quad 93\end{array}\right)$ and histogram of compressional $\Psi_{\mathrm{C}}$ (c) and shear $\Psi_{\mathrm{S}}$ contribution of the INM modes of the mobile inner particles of the hard-wall confined $N \quad 105(70)$ dipole cluster $\left(\begin{array}{ll}\Gamma & 80\end{array}\right)$ as a function of the INM frequencies. Darker colours indicate higher abundance of modes. Both clusters show a comparable histogram. The different $y$-scale results from different normalizations. Higher abundance is found mainly for $\Psi_{\mathrm{C}}$ for both types of confinement.

than that captured by eqn (9) and (10). However, from Fig. 4 it is seen that the modes of the analysed dipole clusters seem to have a stronger compressional character. Since one can not distinguish angular and radial modes from the histograms of $\Psi_{\mathrm{c}}$ and $\Psi_{\mathrm{s}}$ alone, the nature of angular and radial modes for both types of investigated confinement will be discussed in the next section.

\section{Radial and angular modes}

Derivation of the radial and angular eigenmodes requires a decomposition of the dynamical matrix, eqn (5), in a radial and an angular part. In polar coordinates $(r, \theta)$, the dynamical matrix can be rewritten as a block matrix of the form ${ }^{38}$

$$
H(t)=\left(\begin{array}{ll}
H_{r r}(t) & H_{r \theta}(t) \\
H_{\theta r}(t) & H_{\theta \theta}(t)
\end{array}\right)
$$

The sub-matrices contain purely radial modes $H_{r r}(t)$, purely angular modes $H_{\theta \theta}(t)$ as well as mixed radial-angular modes $H_{r \theta}(t)$ and $H_{\theta r}(t)$. The elements of the sub-matrices are given as

$$
H_{r_{i} r_{j}}(t)=\frac{\partial^{2} E(\vec{r}, t)}{\partial r_{i}, \partial r_{j}}
$$

for the radial Hessian $H_{r r}(t)$, 


$$
H_{\theta_{i} \theta_{j}}(t)=\frac{1}{r_{i} r_{j}} \frac{\partial^{2} E(\vec{r}, t)}{\partial \theta_{i}, \partial \theta_{j}}
$$

for the angular Hessian $H_{\theta \theta}(t)$ and

$$
H_{r \theta}(t)=\left(\begin{array}{l}
\frac{1}{r_{i}} \frac{\partial^{2} E(\vec{r}, t)}{\partial \theta_{i}, \partial r_{i}} \frac{1}{r_{j}} \frac{\partial^{2} E(\vec{r}, t)}{\partial r_{i}, \partial \theta_{j}} \\
\frac{1}{r_{i}} \frac{\partial^{2} E(\vec{r}, t)}{\partial \theta_{i}, \partial r_{j}} \frac{1}{r_{j}} \frac{\partial^{2} E(\vec{r}, t)}{\partial r_{j}, \partial \theta_{j}}
\end{array}\right)
$$

for the mixed Hessian $H_{r \theta}(t)$, accordingly. Symmetry of the full Hessian is ensured via $H_{\theta r}(t)=H_{r \theta}(t)^{T}$. First-order derivatives occurring in $H_{r \theta}(t)$ and $H_{\theta r}(t)$ are neglected due to the fact that the system is assumed to be in equilibrium.

We evaluated the density of states for the purely radial and angular eigenmodes for all clusters in both types of confinement. Accordingly, one can calculate separately the radial and angular hopping rate and diffusion constant, see eqn (7) and (8) relying on the angular and radial eigenmodes. However, the spectra itself might be affected by the used coordinate system since the INM eigenfrequencies depend on the chosen coordinate basis, too. ${ }^{51}$

Fig. 5 shows the radial and angular eigenmodes for a parabolically trapped $N=71$ and a hard-wall confined $N=105(70)$ colloidal cluster at three different coupling strength $I$. The coupling strength was varied by changing the magnetization of the particles via tuning the magnetic field of the Helmholtz coils.

The coupling strength of the parabolically confined cluster, see Fig. 5(a) and (b), changes between 142 and 93, whereas the coupling strength varies between 465 and 80 for the hard-wall case as sketched in Fig. 5(c) and (d), thus covering a wider range of coupling. This can be understood from the definition of $\Gamma$, see eqn (1): in the case of hard-wall confinement, the trap is fixed in size and the particles in the cluster do not feel external, inward directed restoring forces $\vec{F}_{\mathrm{ext}, i}^{\mathrm{hw}}=\nabla V_{\mathrm{ext}}^{\mathrm{hw}}=0$ as in the case of the parabolic potential trap where $\vec{F}_{\mathrm{ext}, i}^{\text {para }}=\nabla V_{\mathrm{ext}}^{\mathrm{para}}=$ $m \omega_{0}{ }^{2} \vec{r}_{i}$. Consequently, the density $n$ of the system, which enters in eqn (1) via $b_{\mathrm{ws}}$, does not change when altering the interaction strength $M$ if the particles are trapped in a hard-wall compartment. Otherwise, when lowering the interaction strength of the colloids in a parabolic potential, also the particle density increases due to the persistent restoring forces of the trap that push the particles together into the centre of the confinement. ${ }^{13}$

The DOS for the radial eigenmodes of the $N=71$ cluster in the parabolic trap is shown in Fig. 5(a). Stable modes are excited up to normalized frequencies of roughly 12 for the highest coupling strength $(\Gamma=142)$, unstable modes up to a normalized frequency of 5 . A few peaked features are seen in the stable and unstable mode branches reflecting the localized particle dynamics. When lowering the coupling strength, modes are excited up to higher stable and unstable frequencies. In addition, the peaked structure disappears and the density of states of the radial eigenmodes becomes almost structureless at the lowest coupling strength of $\Gamma=93$. A similar behaviour was
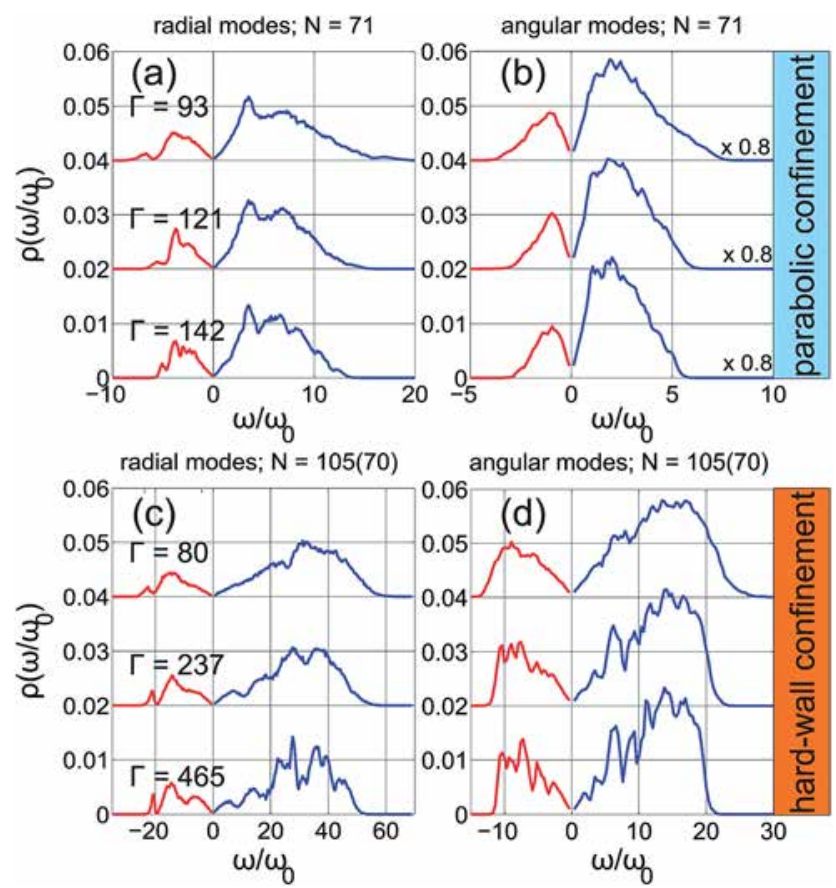

Fig. 5 (a) Radial modes and (b) angular modes for a parabolically confined $N 71$ cluster for different coupling strength. (c) Radial modes and (d) angular modes for a hard-wall confined $N$ 105(70) cluster. For better visibility, the spectra are plotted with a constant offset and multiplied by a constant factor in (b). Lowering the coupling strength leads to diminishing of peaked structures in the mode spectra. Note the different frequency axis for angular and radial eigenmodes in both types of confinement.

found previously for Lennard-Jones clusters and finite 2D dust clusters in plasmas. ${ }^{37,39}$ The lower the coupling strength, the higher the amount of fluctuations of the particle positions within time, see ref. 20 and Section 6. Hence, the positions of the cluster particles are not as localized as for high coupling and slight changes in the particle positions already change the eigenfrequencies calculated from INM. Consequently, the mode spectra get blurred.

The evolution of the angular eigenmodes for the parabolically trapped $N=71$ dipole cluster can be seen from Fig. 5(b). Contrary to the radial modes, stable angular modes are excited up to a normalized frequency of 6 for the highest coupling strength. The unstable angular mode branch does not show a well defined structure for all coupling strengths. The maximum in the density of states for the stable angular modes is found at $\omega / \omega_{0} \approx 1.9$ regardless of the coupling strength. Again as for the radial modes, lowering the coupling strength leads to the excitations of modes at higher frequencies.

The spectra of radial eigenmodes under hard-wall confinement and for $N=105(70)$ as a function of the coupling strength are depicted in Fig. 5(c). Modes are excited up to higher normalized frequencies of about 50. As before, for the highest coupling strength, the corresponding mode spectra shows sharp peaks in both, stable and unstable, regimes. With lowering the coupling strength, the radial mode spectra become 
more and more structureless as for the parabolic case and like in ref. 37 and 39.

A similar behaviour is seen for the spectra of the angular hard-wall modes of a $N=105(70)$ cluster shown in Fig. 5(d). Here and in contrast to the parabolically confined clusters, several peaks are found at high coupling strength. Again, the spectra become blurred when coupling is lowered. Instead of the parabolically confined cluster, the maximum of the DOS of the stable angular modes is shifted to the high frequency part. Contrary to the radial modes, angular modes are excited up to a normalized frequency of roughly 20. For the lowest coupling strength achieved in the experiment, $\Gamma=80$, the highest contribution in the stable part of the density of states is found at $\omega / \omega_{0}=32$ for the radial and roughly 17 for the angular modes. These are exactly the low and high frequency regimes for the overall density of states calculated from the full Hessian (eqn (5)) of the $N=105(70)$ dipole cluster highlighted in the middle row of Fig. 3(b). For the corresponding parabolic case, radial and angular modes are excited in the same frequency region.

How can the splitting of angular and radial modes for dipole clusters be interpreted? In classical confined systems, less energy is necessary to excite angular modes compared to radial modes due to the strong radial constraints. ${ }^{15,55,57}$ As a consequence, angular modes are less stable than radial modes and more unstable angular modes should be excited than unstable radial modes at the same energy level. We have observed for our colloidal dipole clusters that the fraction of unstable angular modes $f_{\mathrm{u}, \theta}=\int_{0}^{-\infty} \rho_{\mathrm{u}, \theta}(\omega) \mathrm{d} \omega$ is always higher than the fraction of unstable radial modes $f_{\mathrm{u}, r}=\int_{0}^{-\infty} \rho_{\mathrm{u}, r}(\omega) \mathrm{d} \omega$. For the systems presented here, on average, one finds $f_{\mathrm{u}, r} \approx 17.4 \%$ and $f_{\mathrm{u}, \theta} \approx$ $19.4 \%$ for the parabolically confined $N=71$ cluster and $f_{\mathrm{u}, r} \approx$ $16.4 \%$ and $f_{\mathrm{u}, \theta} \approx 23.3 \%$ for the hard-wall trapped $N=105(70)$ cluster, confirming the above mentioned arguments.

The sensitivity of angular modes to instabilities was previously observed for finite $2 \mathrm{D}$ dust clusters that interact via the screened Coulomb potential. ${ }^{56,58}$ However, in finite dust clusters, the inter-particle spacing exceeds by far the grain diameter ( $b_{\mathrm{Ws}} \gg a$ ) validating the assumption of a point-charge model. Instead, for our colloidal clusters, the Wigner-Seitz distance is of comparable magnitude $\left(b_{\mathrm{Ws}} \approx a\right)$. Thus, the finite size of each individual colloidal particle should influence the dynamics of the cluster, especially when the packing fraction $\Phi=\left(a / b_{\mathrm{Ws}}\right)^{2}$ exceeds a critical value. The impact of $\Phi$ on the transport properties of the clusters will be discussed in Section 7 .

\section{Inter-particle distance fluctuations}

In INM, the fluid-like behaviour of a system is related to the unstable modes housed in $\rho_{\mathrm{u}}(\omega)$. An alternative way to describe the onset of fluid-like behaviour is often in terms of Lindemann-like parameters. ${ }^{5,59}$ In finite systems, the so-called interparticle distance fluctuations (IDFs) are used instead. ${ }^{60,61}$ To emphasize the role of the coupling strength and the packing fraction onto the particle fluctuations, we calculate the relative IDF via the definition used in ref. 61 and 62

$$
u_{\mathrm{rel}}=\frac{2}{N(N \quad 1)} \sum_{1 \leq i<j}^{N} \sqrt{\frac{\left\langle r_{\mathrm{ij}}{ }^{2}\right\rangle}{\left\langle r_{i j}\right\rangle^{2}}} \quad 1,
$$

with $N$ being the total number of particles in the ensemble and $r_{i j}$ being the distance between particles $i$ and $j$.

Fig. 6 shows the IDF values obtained via eqn (15) for both types of confinement as a function of the coupling strength and the packing fraction. In the case of parabolic confinement, see Fig. 6(a) and (b), the IDFs are grouped in two populations, either for small clusters with $N \leq 16$ or for larger clusters with $N>16$. The smaller dipole clusters show a larger scatter in the data. Here, except one outlier, IDF values of roughly $7-12 \%$ are measured for $N \leq 16$ whereas IDF values of about $3-6 \%$ are found for the population of larger parabolically trapped dipole clusters with $N>16$. For comparison, if $u_{\text {rel }}$ exceeds $18 \%$, 3D Yukawa clusters are said to be in the molten state. ${ }^{62}$ Hence, the observed dipole clusters are well in the solid regime, confirming the visual impression of Fig. 2. For both populations, fluctuations decrease with increasing coupling strength. This stands in contrast to the behaviour of the fluctuations as a function of the packing fraction. Here, one observes a strongly increasing trend for the small clusters with $N \leq 16$ and almost no dependence for the larger clusters with increasing $\Phi$. Again, this can be

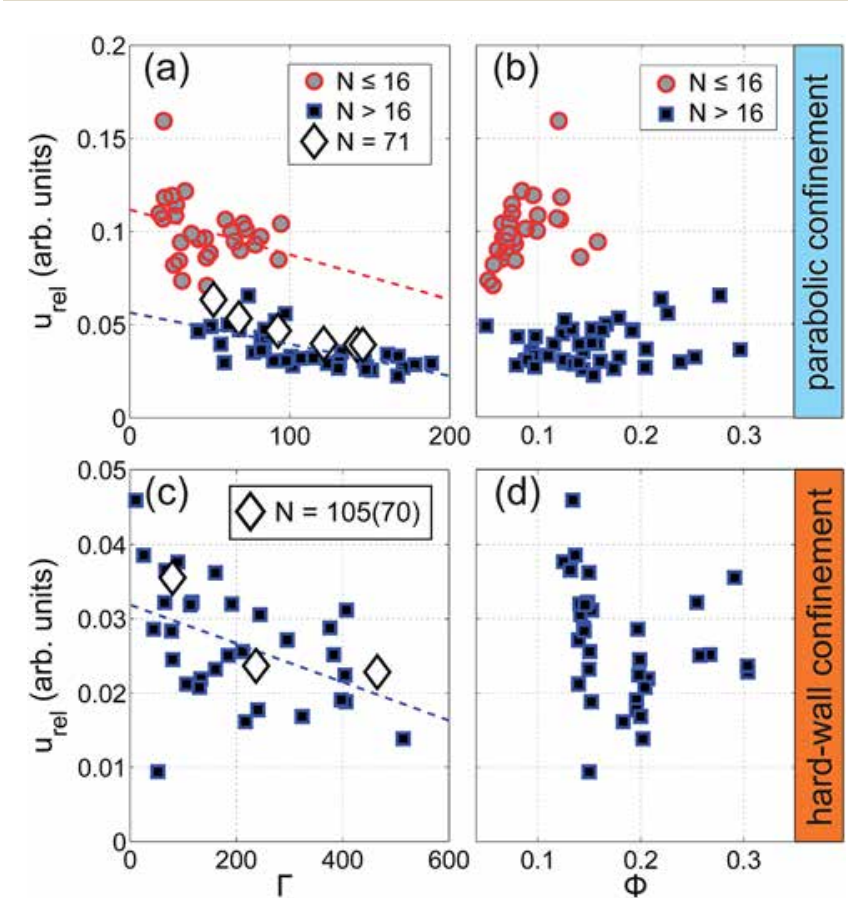

Fig. 6 (a) Inter-particle distance fluctuations $u_{\text {rel }}$ as a function of the coupling strength and (b) as a function of the packing fraction for parabolically confined clusters. (c) IDFs $u_{\text {rel }}$ as a function of the coupling strength and (d) as a function of the packing fraction for hardwall trapped clusters. For the parabolic case, the IDFs are grouped for small clusters with $N \leq 16$ and larger clusters with $N>16$. In (a) and (c), the dashed lines are to guide the eye and the $u_{\text {rel }}$ results for the parabolically trapped $N \quad 71$ and the hard-wall confined $N$ 105(70) cluster are added as a special example. 
attributed to the complex interplay between mutual particle interaction and external confining force.

The inter-particle distance fluctuations for hard-wall trapped clusters are depicted in Fig. 6(c) and (d). Again, fluctuations decrease with increasing coupling strength from about $2 \%$ at high coupling strength to about $4.5 \%$ at very low coupling. In contrast to the previous case of parabolic trapping, no clear trend is observed for the relative inter-particle distance fluctuations with increasing packing fraction. For the hard-wall trapped systems, the packing fraction is calculated using the inner particles and thus $\Phi$ might slightly change upon variation of the coupling strength.

The $u_{\text {rel }}$ values for the parabolically confined $N=71$ cluster decrease from roughly $6.3 \%$ at low coupling strength to $3.9 \%$ at high coupling. For the hard-wall trapped $N=105(70)$ cluster, a decrease from $3.5 \%$ to $2.2 \%$ is observed as the coupling strength is lowered. Even though the change in $u_{\text {rel }}$ seems negligible, fluctuations have a strong impact on the spectral properties, as discussed in the previous section. In this respect, fluctuations can be seen as a precursor of fluid-like behaviour.

\section{Transport coefficient from INM}

We have calculated the diffusion coefficient on the basis of the INM technique for various dipole clusters in parabolic and hard-wall potentials. Typical results are shown in Fig. 7. The relative error in calculating the diffusion coefficient via INM was found to be up to $11 \%$. For all clusters analysed via INM, the resulting diffusion coefficient stays nearly constant over the entire range of coupling achieved in the experiment. This is the expected behaviour for the short-time diffusion coefficient accessible via INM. Additionally, most observed clusters had a coupling parameter that would refer to the solid state of a corresponding extended crystal. ${ }^{5}$ In this phase, the diffusion coefficient of a colloidal dipole crystal scales only weakly with $\Gamma$, whereas significant differences between angular and radial diffusion were found for clusters in hard-wall pots at low $T .^{25,45}$

In Fig. $7(\mathrm{a})$, angular diffusion $D_{\theta}^{\text {para }}(N=71) \approx\left(\begin{array}{ll}3.3 & 4.2\end{array}\right) \times$ $10^{-9} \mathrm{~m}^{2} \mathrm{~s}^{-1}$ is found higher than radial diffusion $D_{r}^{\text {para }}(N=71)$ $\approx 1 \times 10^{-9} \mathrm{~m}^{2} \mathrm{~s}^{-1}$ for the parabolically confined $N=71$ cluster. In this particular example, both angular and radial diffusion tend to higher values as the coupling parameter falls below a critical value of roughly $\Gamma=68$ which is close to the melting point for extended dipole crystals. ${ }^{7}$ Such a behaviour could not be seen for all investigated clusters.

Fig. 7(b) shows the angular and radial diffusion coefficient for the hard-wall trapped $N=105(70)$ cluster. Angular and radial diffusion are smaller than for the parabolic cluster, reaching values of about $D_{\theta}^{\mathrm{hw}}(N=105(70)) \approx\left(\begin{array}{ll}7 & 8.5\end{array}\right) \times 10^{-11}$ $\mathrm{m}^{2} \mathrm{~s}^{-1}$ and $D_{r}^{\mathrm{hw}}(N=105(70)) \approx\left(\begin{array}{ll}2 & 3.8\end{array}\right) \times 10^{-11} \mathrm{~m}^{2} \mathrm{~s}^{-1}$. In general, diffusion in hard-wall traps is found to be roughly one to two orders of magnitudes lower than for the parabollically confined dipole ensembles. It seems that both radial and angular diffusion coefficients have a slightly increasing trend as the coupling strength increases. Such a behaviour was found for most of the analysed hard-wall confined clusters. This can be understood by the fact that with increasing coupling parameter,
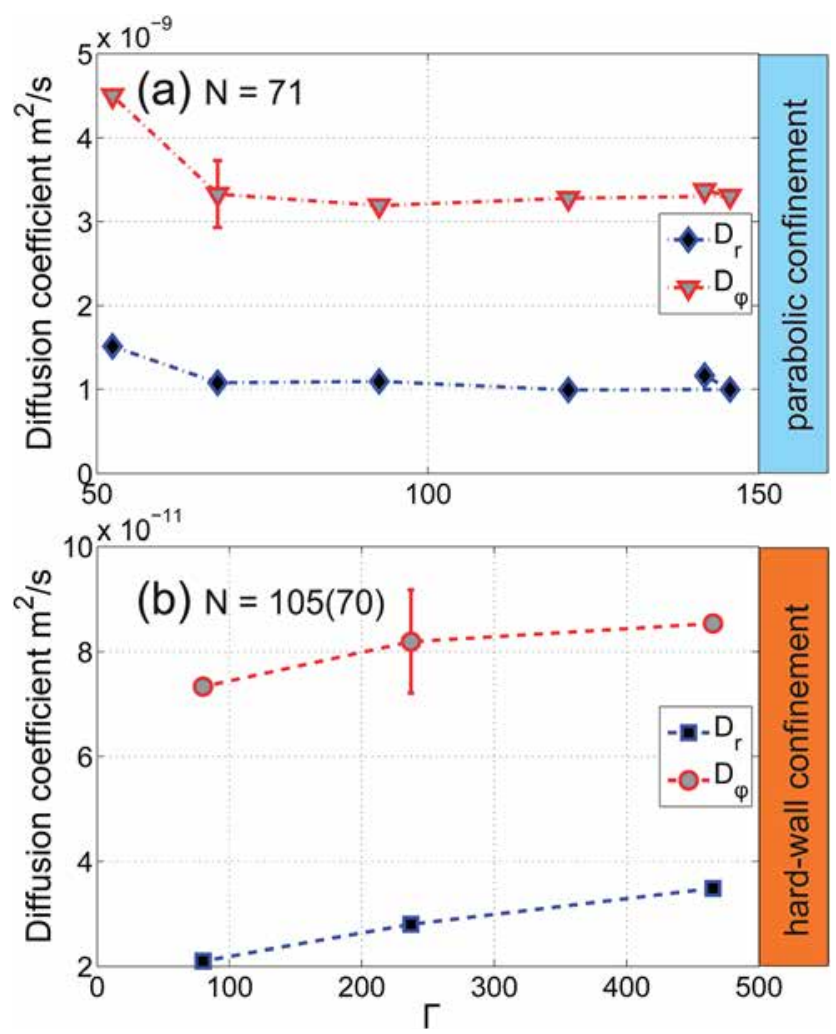

Fig. 7 (a) Diffusion coefficients as function of coupling strength for the parabolically confined $N 71$ cluster and (b) for the hard-wall trapped N 105(70) dipole cluster. Typical uncertainties for estimating the diffusion coefficients are added as error bars. Note that diffusion is about one to two orders of magnitude higher in the parabolic trap.

each particle undergoes more vivid positional fluctuations due to interactions with nearby particles which then lead to an increased effective diffusion coefficient. Because in case of the parabolic confinement particles can escape laterally with increasing $\Gamma$, the increase of the diffusion coefficient is less pronounced under such conditions (Fig. 7).

At high densities, the size of the individual beads can not be neglected. ${ }^{2,24,26}$ Hence, a higher packing fraction should lead to a lower mobility and hence lower diffusion of the particles. ${ }^{23}$ Zahn et al. pointed out that hydrodynamic interactions may enhance diffusion of the particles in dense suspensions. ${ }^{4}$ Even more complex dependencies on hydrodynamic interactions were found when the particles are subject to periodic potentials. $^{19}$

The diffusion coefficient as a function of the packing fraction is imaged in Fig. 8 for all analysed dipole clusters. The results are shown for the parabolic trap in Fig 8(a). At low packing fraction, $\Phi<0.1$, angular diffusion exceeds the radial diffusion and the values for $D_{\theta}$ and $D_{r}$ have a large scatter. With increasing packing fraction, angular diffusion tends to lower values and converges to the trend of the radial diffusion coefficient at roughly $\Phi \geq 0.2$. Van Megen $e t$ al. investigated the impact of the packing fraction on the short-time diffusion coefficient of hardsphere colloids., ${ }^{3,24}$ They have revealed that the self-diffusion coefficient tends to lower values as the volume fraction 


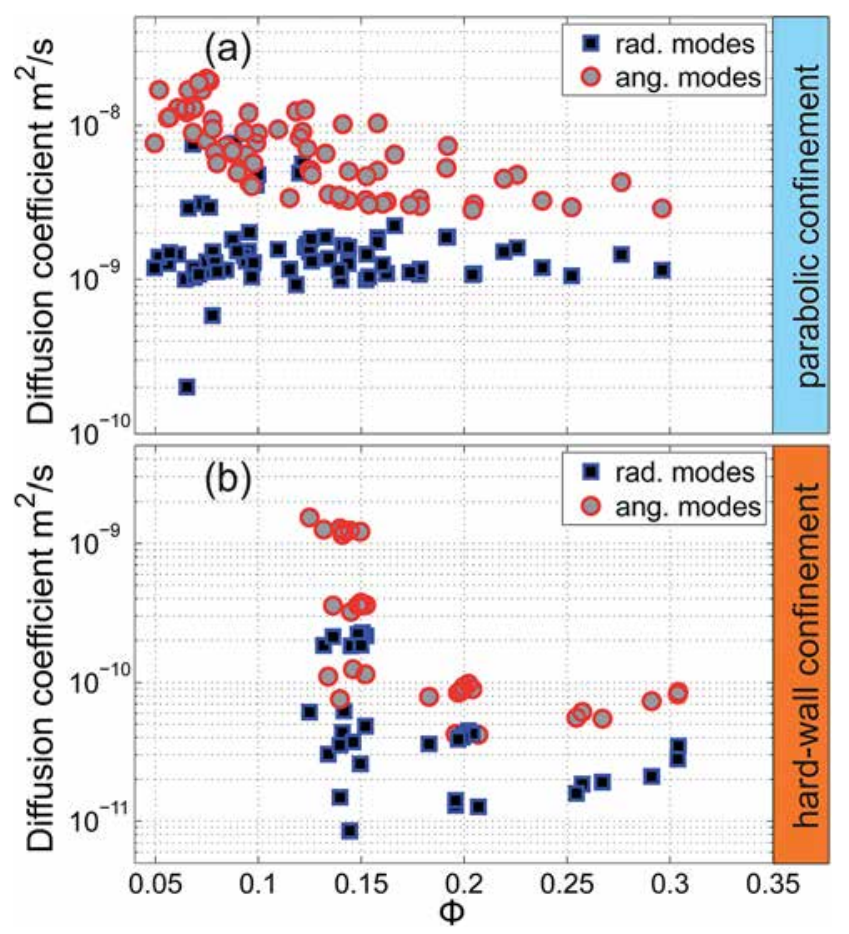

Fig. 8 (a) Diffusion coefficients as function of the packing fraction $\Phi$ for parabolically and (b) for hard-wall confined dipole clusters. With increasing packing fraction, angular diffusion converges with radial diffusion for particles in parabolic traps.

increases, which seems to be the case for the dipole interacting particles in parabolic traps, too. As already noted, the parabolic trap leads to restoring forces of each individual particle. With decreasing magnetic field strength, i.e. diminishing dipole interaction between the beads, the grains sediment to the "bottom" of the potential trap. There, the packing fraction is highest and the particle motion seems to be collective and "glassy". ${ }^{10,52}$

In the case of hard-wall confinement the angular diffusion has again the tendency to be higher than the radial diffusion in the range of the accessible packing fractions, see Fig. 8(b). When the packing fraction exceeds the value $\Phi=0.17$, the angular diffusion coefficient drops one order of magnitude.

However, one should bear in mind that also the exact particle number might alter the diffusive properties since finite systems always show a competition between surface and volume effects. ${ }^{15,63,64}$ To hint at these, the radial and angular diffusion coefficients as a function of the particle number are depicted in Fig. 9. The diffusion coefficients of the parabolically confined clusters, see Fig. 9(a), in general decrease with increasing particle number. Additional effects are seen at particle numbers below $N \leq 16$ where the diffusion coefficients have a larger scatter and are generally higher. This is the regime where the fluctuations $u_{\text {rel }}$ were found higher and the mode spectra showed a pronounced, peaked structure due to discrete eigenmodes. Angular diffusion changes much stronger with the particle number and tends to lower values as the particle number increases. Moreover, the distance in magnitude between angular and radial diffusion shrinks with increasing
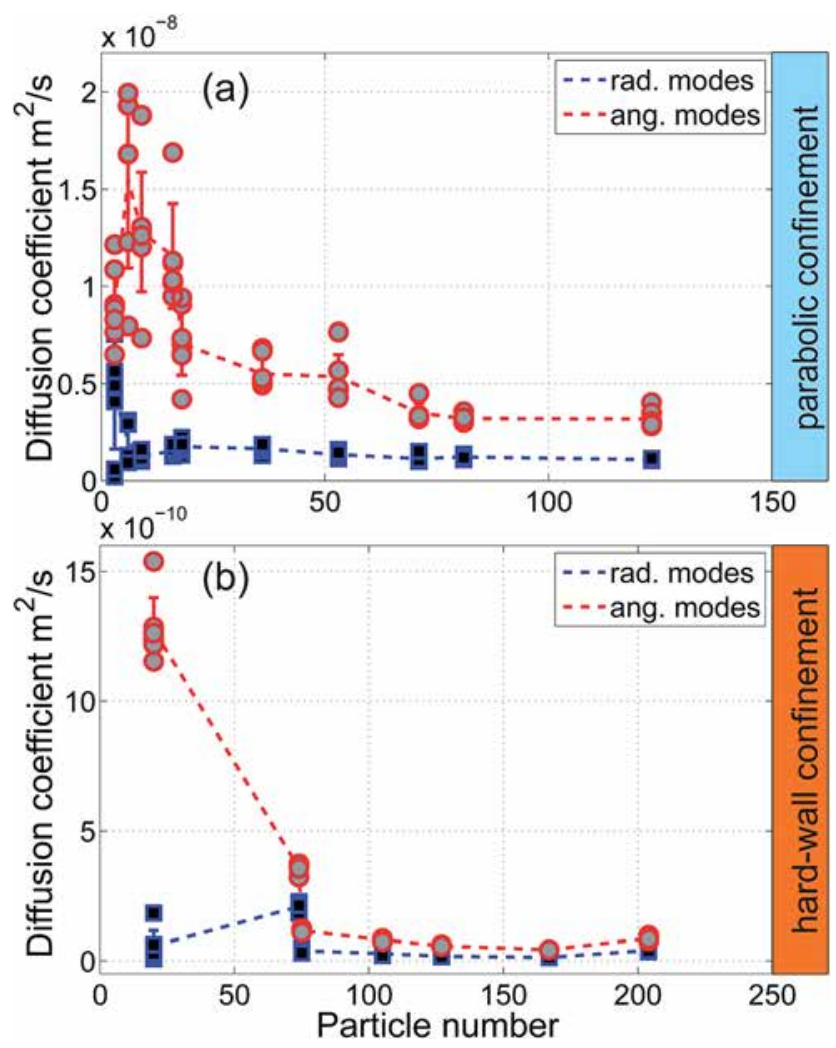

Fig. 9 (a) Angular and radial diffusion coefficient as function of particle number for parabolically confined clusters. At low particle numbers, finite size effects become important. When the particle number is increased, the distance between angular and radial diffusion shrinks. (b) Angular and radial diffusion coefficient as function of particle number for hard-wall trapped clusters. For very large clusters with $N>$ 100, radial and angular diffusion are no longer separated and the ensembles behave like bulk material. To guide the eye, the mean angular and radial diffusion coefficients obtained when averaging over $\Gamma$ for each cluster are added as dashed lines together with the corresponding error bars.

system size. Again, this confirms that the dynamical behaviour of a finite ensemble is influenced by the exact particle number. ${ }^{15,20}$ Nelissen et al. ${ }^{54}$ found that a transition to bulk-like behaviour for Coulomb clusters in parabolic traps takes place in the region between 120 and 200 particles.

Angular and radial diffusion coefficients as a function of the particle number of the hard-wall confined clusters are sketched in Fig. 9(b). Due to the lower number of investigated dipole ensembles especially at low particle number, no statement can be given concerning the finite size fluctuations, here. However, a the gap between radial and angular diffusion that was seen for parabolically clusters diminishes here when the ensembles are larger than roughly $N>100$. Hence, neither angular nor radial directed motion is "preferred", as in the case of small systems, ${ }^{12,13}$ and the larger dipole clusters already behave like extended monolayers. This picture is fully consistent with the previous findings and moreover shows the impact of the external confinement on the diffusive properties of the particles. 


\section{Conclusions}

Dynamical properties of finite 2D colloidal clusters were presented. The particles were trapped either in parabolic or hardwall confinement. The dipole interaction of the paramagnetic beads was varied using an external magnetic field. The method of instantaneous normal modes was introduced for colloidal dipole clusters. A density of states for the normal modes was derived from long-run time series by using the instant particle configurations as an input. Small clusters show a discrete mode spectra for stable and unstable modes. With increasing size of the system, the mode peaks in the spectra diminish. For hardwall confined clusters, two groups of stable high and lowfrequency modes are found even for large clusters, whereas the DOS becomes almost structureless in the case of large ensembles in parabolic confinement. With decreasing interaction strength, a peaked mode structure of the confined clusters diminishes due to enhanced fluctuations in the particle configurations. For both types of confinement, angular modes are excited at lower frequencies than the radial modes.

The diffusive properties of the system have been calculated from the unstable modes governed by the INM technique. Radial and angular diffusion have been revealed using only radial and angular modes. In general, diffusion is one to two orders of magnitude higher for parabolically confined systems. No pronounced dependence of the short-time diffusion coefficient with the coupling strength was found. In contrast, mainly angular diffusion is altered with increasing packing fraction and tends to lower values.

The exact particle number dominates the diffusive properties primarily at low particle numbers and in the case of parabolic confinement. As the system size increases, the difference in magnitude between radial and angular diffusion shrinks. Due to the lack of statistics, no statement can be given for hardwall confinement concerning the finite size effects. Contrary to the parabolic case, the gap between radial and angular diffusion diminishes in the limit of high particle numbers, hinting toward bulk behaviour of the large hard-wall trapped clusters.

\section{Acknowledgements}

Financial support by the Deutsche Forschungsgemeinschaft via SFB-TR24 Grant A3 is gratefully acknowledged.

\section{References}

1 R. A. L. Jones, Soft Condensed Matter, Oxford Unversity Press, 2002.

2 A. Ivlev, H. Löwen, G. Morfill and C. P. Royall, Complex Plasmas and Colloidal Dispersions: Particle-Resolved Studies of Classical Liquids and Solids (Series in Soft Condensed Matter), World Scientific Pub Co, 2012.

3 W. van Megen and S. M. Underwood, J. Chem. Phys., 1989, 91, 552-559.

4 K. Zahn, J. M. Méndez-Alcaraz and G. Maret, Phys. Rev. Lett., 1997, 79, 175-178.
5 K. Zahn, R. Lenke and G. Maret, Phys. Rev. Lett., 1999, 82, 2721-2724.

6 P. Keim, G. Maret, U. Herz and H. H. von Grünberg, Phys. Rev. Lett., 2004, 92, 215504.

7 P. Keim, G. Maret and H. H. von Grünberg, Phys. Rev. E: Stat., Nonlinear, Soft Matter Phys., 2007, 75, 031402.

8 S. Deutschländer, T. Horn, H. Löwen, G. Maret and P. Keim, Phys. Rev. Lett., 2013, 111, 098301.

9 D. Kaya, N. L. Green, C. E. Maloney and M. F. Islam, Science, 2010, 329, 656-658.

10 A. Ghosh, R. Mari, V. Chikkadi, P. Schall, J. Kurchan and D. Bonn, Soft Matter, 2010, 6, 3082-3090.

11 A. Ghosh, V. K. Chikkadi, P. Schall, J. Kurchan and D. Bonn, Phys. Rev. Lett., 2010, 104, 248305.

12 R. Bubeck, C. Bechinger, S. Neser and P. Leiderer, Phys. Rev. Lett., 1999, 82, 3364-3367.

13 I. Schweigert, V. A. Schweigert and F. Peeters, Phys. Rev. Lett., 2000, 84, 4381.

14 M. Kong, B. Partoens and F. M. Peeters, New J. Phys., 2003, 5, 23.

15 M. Kong, B. Partoens, A. Matulis and F. M. Peeters, Phys. Rev. E: Stat., Nonlinear, Soft Matter Phys., 2004, 69, 036412.

16 S. Apolinario, B. Partoens and F. M. Peeters, Phys. Rev. E: Stat., Nonlinear, Soft Matter Phys., 2005, 72, 046122.

17 K. Mangold, J. Birk, P. Leiderer and C. Bechinger, Phys. Chem. Chem. Phys., 2004, 6, 1623-1626.

18 W. Yang, K. Nelissen, M. Kong, Y. Li and Y. Tian, Eur. Phys. J. $B, 2011,83,499-505$.

19 E. C. Euán-Díaz, V. R. Misko, F. M. Peeters, S. HerreraVelarde and R. Castañeda Priego, Phys. Rev. E: Stat., Nonlinear, Soft Matter Phys., 2012, 86, 031123.

20 T. O. E. Skinner, H. M. Martin, D. G. A. L. Aarts and R. P. A. Dullens, Soft Matter, 2013, 9, 10586-10591.

21 N. Vandewalle, N. Obara and G. Lumay, Eur. Phys. J. E: Soft Matter Biol. Phys., 2013, 36, 127.

22 S. Tanaka, Y. Oki and Y. Kimura, Phys. Rev. E: Stat., Nonlinear, Soft Matter Phys., 2014, 89, 052305.

23 G. L. Hunter, K. V. Edmond and E. R. Weeks, Phys. Rev. Lett., 2014, 112, 218302.

$24 \mathrm{~W}$. van Megen, S. M. Underwood, R. H. Ottewill, N. S. J. Williams and P. N. Pusey, Faraday Discuss. Chem. Soc., 1987, 83, 47-57.

25 R. Bubeck, S. Neser, C. Bechinger and P. Leiderer, Trends in Colloid and Interface Science XII, Steinkopff, 1998, vol. 110, pp. 41-45.

26 G. L. Hunter and E. R. Weeks, Rep. Prog. Phys., 2012, 75, 066501.

27 A. Melzer, A. Schella, J. Schablinski, D. Block and A. Piel, Phys. Rev. E., 2013, 87, 033107.

28 O. S. Vaulina, X. G. Adamovich, O. F. Petrov and V. E. Fortov, Phys. Rev. E, 2008, 77, 066404.

29 G. Seeley and T. Keyes, J. Chem. Phys., 1989, 91, 5581-5586.

30 R. M. Stratt, Acc. Chem. Res., 1995, 28(5), 201-207.

31 S. D. Bembenek and B. B. Laird, Phys. Rev. Lett., 1995, 74, 936-939.

32 T. Keyes, J. Chem. Phys., 1994, 101(6), 5081-5092.

33 G. V. Vijayadamodar and A. Nitzan, J. Chem. Phys., 1995, 103(6), 2169-2177. 
34 T. Keyes, J. Phys. Chem. A, 1997, 101, 2921-2930.

35 J. D. Gezelter, E. Rabani and B. J. Berne, J. Chem. Phys., 1997, 107(12), 4618-4627.

36 E. La Nave, A. Scala, F. W. Starr, F. Sciortino and H. E. Stanley, Phys. Rev. Lett., 2000, 84, 4605-4608.

37 J. E. Adams and R. M. Stratt, J. Chem. Phys., 1990, 93, 1332-1346.

38 S. Cocco and R. Monasson, J. Chem. Phys., 2000, 112, 1001710033.

39 A. Melzer, A. Schella, J. Schablinski, D. Block and A. Piel, Phys. Rev. Lett., 2012, 108, 225001.

40 A. Melzer, A. Schella, J. Schablinski, D. Block and A. Piel, Phys. Rev. E: Stat., Nonlinear, Soft Matter Phys., 2013, 87, 033107.

41 A. Schella, M. Mulsow, A. Melzer, J. Schablinski and D. Block, Phys. Rev. E: Stat., Nonlinear, Soft Matter Phys., 2013, 87, 063102.

42 V. Blickle, D. Babic and C. Bechinger, Appl. Phys. Lett., 2005, 87, 101102.

43 H. Kählert and M. Bonitz, Phys. Rev. Lett., 2010, 104, 015001.

44 J. Z. Y. Chen, Soft Matter, 2013, 9, 10921-10930.

45 R. Bubeck, P. Leiderer and C. Bechinger, Trends in Colloid and Interface Science XV, Springer Berlin Heidelberg, 2001, vol. 118, pp. 73-76.

46 SU-8 2000.5-2015 Data Sheet, http://www.microchem.com/ Prod-SU82000.htm.

47 G. Fonnum, C. Johansson, A. Molteberg, S. Mrup and E. Aksnes, J. Magn. Magn. Mater., 2005, 293, 41-47.

48 J. C. Crocker and D. G. Grier, J. Colloid Interface Sci., 1996, 179, 298-310.

49 J. K. G. Dhont, An Introduction to Dynamics of Colloids (Studies in Interface Science), Elsevier Science \& Technology, 1996.
50 W.-X. Li, T. Keyes, R. L. Murry and J. T. Fourkas, J. Chem. Phys., 1998, 109, 9096-9100.

51 R. L. Murry, J. T. Fourkas, W.-X. Li and T. Keyes, J. Chem. Phys., 1999, 110, 10410-10422.

52 S. P. Das, Rev. Mod. Phys., 2004, 76, 785-851.

53 A. Melzer, A. Schella and M. Mulsow, Phys. Rev. E: Stat., Nonlinear, Soft Matter Phys., 2014, 89, 013109.

54 K. Nelissen, A. Matulis, B. Partoens, M. Kong and F. M. Peeters, Phys. Rev. E: Stat., Nonlinear, Soft Matter Phys., 2006, 73, 016607.

55 V. A. Schweigert and F. Peeters, Phys. Rev. B: Condens. Matter Mater. Phys., 1995, 51, 7700.

56 A. Melzer, Phys. Rev. E: Stat., Nonlinear, Soft Matter Phys., 2003, 67, 016411.

57 V. M. Bedanov and F. M. Peeters, Phys. Rev. B: Condens. Matter Mater. Phys., 1994, 49, 2667.

58 M. Klindworth, A. Melzer, A. Piel and V. Schweigert, Phys. Rev. B: Condens. Matter Mater. Phys., 2000, 61, 8404.

59 V. Bedanov, G. Gadiyak and Y. Lozovik, Phys. Lett. A, 1985, 109, 289-291.

60 S. W. S. Apolinario and F. Peeters, Phys. Rev. E: Stat., Nonlinear, Soft Matter Phys., 2007, 76, 031107.

61 J. Böning, A. Filinov, P. Ludwig, H. Baumgartner, M. Bonitz and Y. E. Lozovik, Phys. Rev. Lett., 2008, 100, 113401.

62 A. Schella, T. Miksch, A. Melzer, J. Schablinski, D. Block, A. Piel, H. Thomsen, P. Ludwig and M. Bonitz, Phys. Rev. E: Stat., Nonlinear, Soft Matter Phys., 2011, 84, 056402.

63 W.-T. Juan, Z.-H. Huang, J.-W. Hsu, Y.-J. Lai and L. I., Phys. Rev. E: Stat. Phys., Plasmas, Fluids, Relat. Interdiscip. Top., 1998, 58, R6947-R6950.

64 J. P. Schiffer, Phys. Rev. Lett., 2002, 88, 205003. 